\title{
Clinical and Advanced MRI Techniques for Detection of Checkpoint Inhibitor Associated Myocarditis
}

\author{
Amit Arbune $^{1} \cdot$ Aaron Soufer $^{1} \cdot$ Lauren A. Baldassarre ${ }^{1}$
}

Published online: 14 January 2020

(C) The Author(s) 2020

\begin{abstract}
Purpose of Review With the advent of immune checkpoint inhibitors (ICIs), cancer treatment has been revolutionized; however, these agents are associated with immune-related adverse events, including myocarditis, which ranges from mild to fulminant in severity. Currently, there are no established guidelines in diagnosing ICI-associated myocarditis, and the gold standard test for diagnosis of myocarditis in general is invasive endomyocardial biopsy (EMB). Cardiac magnetic resonance (CMR) imaging is a noninvasive test with the advantage of providing structural, functional and tissue characterization information. Additionally, it provides high spatial and temporal resolution without exposure to ionizing radiation, iodinated contrast, or radioactive isotopes. Recent Findings With an increasing number of reported cases of ICI associated myocarditis, understanding of the disease process and associated CMR findings is growing. Diagnostic testing with cardiac biomarkers, electrocardiogram, and echocardiogram can be nonspecific and EMB can have sampling errors. CMR as a diagnostic tool can provide functional assessment of biventricular ejection fraction, myocardial strain, tissue characterization of myocardial edema and inflammation as well as fibrosis. Furthermore, with advanced parametric mapping techniques, CMR provides even more sensitive and quantitative information about myocardial inflammation and fibrosis, including measurements of extracellular volume.

Summary ICI-associated myocarditis is a serious immune adverse event, and CMR plays a vital role in establishing its diagnosis, providing prognostic information, and has the potential for use as a tool for screening and serial monitoring in patients exposed to ICIs.
\end{abstract}

Keywords Cardiac magnetic resonance imaging $\cdot$ Immune checkpoint inhibitor associated myocarditis $\cdot$ Immune checkpoint inhibitor cardiotoxicity $\cdot$ Late gadolinium enhancement $\cdot$ Myocardial edema $\cdot$ Parametric mapping

\section{Introduction}

Treatment with immune checkpoint inhibitors (ICIs) is revolutionizing cancer treatment, but at the same time, these novel therapies are associated with immune-related adverse events (irAEs) affecting almost all organs, which can be serious and life-threatening in nearly $20 \%$ of cases (1-4). These irAEs

Topical Collection on Cardio-Oncology

Lauren A. Baldassarre

lauren.baldassarre@yale.edu

Amit Arbune

amit.arbune@yale.edu

Aaron Soufer

aaron.soufer@yale.edu

1 Department of Cardiovascular Medicine, Yale University School of Medicine, New Haven, CT, USA include significant cardiotoxicity, although the true incidence of cardiovascular complications is unknown. The most commonly reported cardiac irAEs are myocarditis, followed by heart failure with systolic dysfunction, pericarditis, and arrhythmias (3, 5-9). Myocarditis is more common in patients receiving a combination of ICIs compared to a single agent (10). The exact mechanism of this autoimmune myocarditis is not well established but involves disruption of the programmed cell death protein-1 (PD-1) pathway (3). Of note, autoimmune myocarditis has been demonstrated in multiple mouse models with a deficiency in the PD-1 pathway (11). There are currently no guidelines for monitoring or early detection of these cardiac irAEs. Prospective cardiac imaging studies have not been performed in patients receiving these therapies, and therefore, the optimal modality for detection is not known. Cardiac magnetic resonance (CMR) imaging is an invaluable tool in establishing the diagnosis of ICI-associated myocarditis in patients in whom there is a clinical suspicion. 
Early diagnosis and initiation of treatment are critical in these patients, given the fatality associated with the fulminant form of the disease.

\section{Presentation and Diagnosis of $\mathrm{ICl}-$ Associated Myocarditis}

The reported incidence of ICI-associated myocarditis is up to $1.33 \%$ based on case reports, case series, and an adverse event database $(9,10,12,13)$. However, the true incidence may be higher, as no prospective screening studies have been performed. Moreover, it seems that more cases are being recognized in recent years, a trend noted in the retrospective study by Salem et al. (9). Patients can present after the first dose or subsequent doses with a median time to presentation being approximately 1 month after initiation of ICIs $(7-10,13$, 14). The overall incidence of all irAEs is higher with combination anti-cytotoxic $\mathrm{T}$ lymphocyte-associated protein 4 (antiCTLA4) therapy, such as ipilimumab, with anti-PD1 therapy, such as nivolumab and pembrolizumab (15), and this trend holds true for cardiac irAEs as well $(9,10)$. In a case series of 35 patients with ICI-associated myocarditis, Mahmood et al. noted increased incidence in patients who had received combination therapy (10). The incidence of other concomitant irAEs was variable in the patients with ICI-associated myocarditis $(9,10)$, and other risk factors for ICI-associated myocarditis have not yet been well established.

Troponin elevation has been well reported in and is used as a clinical marker for diagnosis and prognosis with myocarditis in general $(16,17)$. However, the troponin elevation depends on the timing of when it is checked in the course of disease and can lack specificity (17). Additionally, the sensitivity of troponin elevation specifically in ICI myocarditis is not yet known. Based on a review of 42 cases of ICI-associated myocarditis, there were 3 cases with no troponin elevation which had an established tissue diagnosis of autoimmune myocarditis with lymphocytic infiltration $(8,12,18,19)$. In a recent study of 76 melanoma patients who had prospective screening with troponin-I for surveillance of ICI-associated myocarditis, no patients developed the disease. However, $17 \%$ had minimally elevated high sensitivity troponin with no other evidence of myocarditis, suggesting a low screening yield for identification of clinical disease, but perhaps suggesting subclinical cardiotoxicity (20). Electrocardiogram (ECG) findings with ICI-associated myocarditis are highly variable, ranging from nonspecific ST segment and T wave changes, ST elevations, and 1 st and 2 nd degree $\mathrm{AB}$ block to complete heart block $(8,21,22)$.

ICI-associated myocarditis has been reported to have variable echocardiographic (echo) findings, ranging from normal LV ejection fraction (LVEF), mildly reduced to severely reduced LVEF, biventricular reduced ejection fraction and regional wall motion abnormalities. In a case series of 35 patients from a multicenter registry with 8 sites, LVEF was normal in $51 \%$ of patients with ICI-associated myocarditis (10). In another review of reported cases of 42 patients of ICI-associated myocarditis, 36\% reported normal EF (8). Additionally, takotsubo-like syndrome as a cardiotoxicity manifestation has been reported in a couple of cases treated with combination ICIs (23).

Endomyocardial biopsy (EMB) is considered the gold standard for diagnosis of myocarditis in general. However, given the sampling error, a negative biopsy does not conclusively rule out myocarditis if there is patchy involvement, as has been noted in viral myocarditis (24). Lymphocytic infiltration with $\mathrm{T}$ cell prominence has been noted on biopsy and some autopsies in patients with ICI-associated myocarditis $(10,18$, 26), though the sensitivity and specificity of biopsy in ICI associated myocarditis has not been defined. Also, being an invasive procedure, it carries a complication rate of $6 \%$, with $0.1-0.5 \%$ of those being serious complications (25). Historically, prior to the advent of ICIs, indications for EMB have been limited to patients with significant heart failure, dangerous arrhythmias, and hemodynamic compromise, making it a nonideal tool for early diagnosis [Cooper 2007]. Guidelines for endomyocardial biopsy to confirm ICIassociated myocarditis are not currently available.

Management of patients with ICI-associated myocarditis has so far been reported to include high-dose glucocorticoids in the majority of patients, with other immunosuppressants such as infliximab or IVIG in used in some steroidrefractory patients, and case reports with the successful use of ATG in patients with fulminant disease $(7,13,14,27-30)$. In general, ICI therapy is discontinued in patients diagnosed with ICI-associated myocarditis, as this is categorized as a high-grade irAE $(4,31)$. Given the limited number of reported cases, the data on reporting of major adverse cardiovascular events (MACE), defined as a composite of cardiovascular death, cardiogenic shock, cardiac arrest, and hemodynamically significant complete heart block, is limited as well. The incidence of MACE has been reported to be fourfold higher

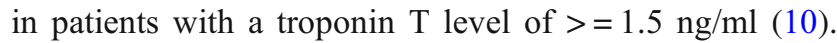
Additionally, in a cohort of ICI-associated myocarditis patients with a normal EF, 38\% still experienced MACE (10).

\section{Role of Cardiac MRI in Assessment of ICl-Associated Myocarditis}

The diagnosis of ICI-associated myocarditis can be challenging as the clinical presentation can range from such symptoms as chest pain and shortness of breath to a presentation of heart failure or fulminant cardiogenic shock $(1,7-10,13,14,19$, $27,32)$. Given this variable clinical presentation, nonspecificity of cardiac biomarkers and ECG changes, variable echocardiogram findings, and the limited indication of $\mathrm{EMB}$, there is a need for a noninvasive diagnostic tool for 
accurate diagnosis. CMR can comprehensively assess biventricular function, regional function, and tissue characterization of inflammation and fibrosis, making it ideal for bridging this need. Furthermore, CMR does not expose patients to ionizing radiation, iodinated contrast, or radioisotope, is not limited by the patient's body habitus, and produces images with high spatial and temporal resolution (33). Most of the cases of ICI-associated myocarditis who underwent CMR had positive diagnostic findings; however, there have been a couple reported cases of CMR without diagnostic findings of myocarditis in this disease state $(7-9,15)$. A recent review by Ganatra and Neilan on ICI-associated myocarditis outlines an algorithm enlisting CMR as a tool to both establish diagnosis in patients and confirm abnormalities found in troponins, NTproBNP, ECG, and echocardiogram (12).

CMR is a well-established noninvasive tool for diagnosing myocarditis in general (33-35). The Lake Louise Criteria were established by the International Consensus Group on CMR diagnosis of Myocarditis for patients with suspected myocarditis (34). The Lake Louise Criteria require 2 or more of the following to establishing the diagnosis of myocarditis: presence of edema, early gadolinium enhancement, and late gadolinium enhancement (LGE). The overall sensitivity with these criteria for diagnosis of myocarditis was noted to be $68 \%$, with a specificity of $91 \%$ and diagnostic accuracy of 78\% (34). In 2018, a scientific expert panel provided an updated consensus recommendation for diagnosis of myocarditis, including the use of parametric mapping techniques, which provide a more quantitative assessment of tissue characterization $(34,35)$. The updated Lake Louise Criteria require at least one T2-based and one T1-based positive finding to establishing the diagnosis of acute myocardial inflammation, with the presence of only one of the two still supportive of the diagnosis (35). Other supporting criteria include evidence of pericarditis and regional or global LV dysfunction $(34,35)$. In general, acute myocarditis tends to have positive findings on both T1- and T2-based imaging, and in more chronic states, such as rheumatologic disease-associated autoimmune myocarditis, these findings can also be seen in a significant number of patients (34).

Pericardial effusion and myopericarditis are reported irAEs with ICIs, occurring less frequently compared to myocarditis alone (9). Additionally, some cases with isolated ICI-associated pericarditis have been reported as well $(5,36)$. CMR can be a very helpful tool for diagnosis of pericarditis, demonstrating a thickened, inflamed, and possibly fibrotic pericardium. At the same time, it can determine presence or absence of ventricular interdependence in cases of suspected constriction. In our retrospective study, we had one case of ICI-associated pericarditis in the setting of a normal LVEF (Fig. 3).

\section{Structural and Functional Analysis}

Patients with ICI-associated myocarditis have been found to have a reduction in their LVEF, isolated RV dysfunction, or biventricular dysfunction, with a varying degree of abnormalities in regional wall abnormalities in some of these patients $(7,8,10,23)$. The role of echocardiography in structural and functional assessment of LV and RV function in patients with myocarditis has been well established $(37,38)$. However, in general, CMR is the gold standard for accurate assessment of left and right ventricular systolic function, providing high spatial resolution without geometric assumptions or limitations from body habitus $(33,39)$. Evaluation of LV and RV function by CMR is preferred using the steady-state free precession (SSFP) cine imaging acquisition, which gives a clear delineation between the myocardium and blood pool $(40,41)$. A contiguous cine SSFP short axis stack (SAS), covering the entire ventricle from base to apex, is obtained perpendicular to the LV long axis with a slice thickness of 6-10 mm, using ECG gating and breath hold technique (33). For assessment of volumes and $\mathrm{EF}$, contouring of the $\mathrm{LV}$ and $\mathrm{RV}$ endocardial borders is performed in both end-diastole and end-systole, either using manual or semiautomatic methods via postprocessing software by Simpson's summation of discs method (Fig. 1). Additionally, standard two-chamber, three-chamber, and four-chamber long axis views also provide information on the structure and regional function of the LV and RV. The functional assessment and volumes obtained by CMR have been validated to be highly reproducible and have low variance on repeated measurement (42).

LV mass can also be accurately measured on CMR using the cine SAS with endocardial and epicardial contours. Papillary muscles and trabeculations can either be included or excluded from the LV mass, as papillary muscle mass accounts for approximately $9 \%$ of LV mass $(43,44)$. There has been no published data on changes in LV mass in ICIassociated myocarditis. However, a transient decrease in LV mass and increase in LV thickness has been observed in patients with myocarditis in general (45). Further research is needed for observing the changes in LV mass in patients with ICI associated myocarditis (Fig. 3).

\section{Myocardial Inflammation Assessment}

T2-weighted imaging on CMR is a non-contrast tissue characterization technique which provides information related to regional or global increases in myocardial water content (33). Native myocardial T2 relaxation times are lengthened by increased water content in inflammatory states, which produces increased signal intensity on T2-weighted images (46). The techniques commonly utilized are breath-held, black blood short-tau inversion recovery sequence (STIR), or T2 prepped SSFP, with either double or triple inversion recovery $(34,35)$. Increased signal intensity on T2-weighted imaging is highly sensitive for the presence of myocardial edema. It is an established criterion for the diagnosis of myocarditis in the original as well as in the updated Lake Louise Criteria (34, 


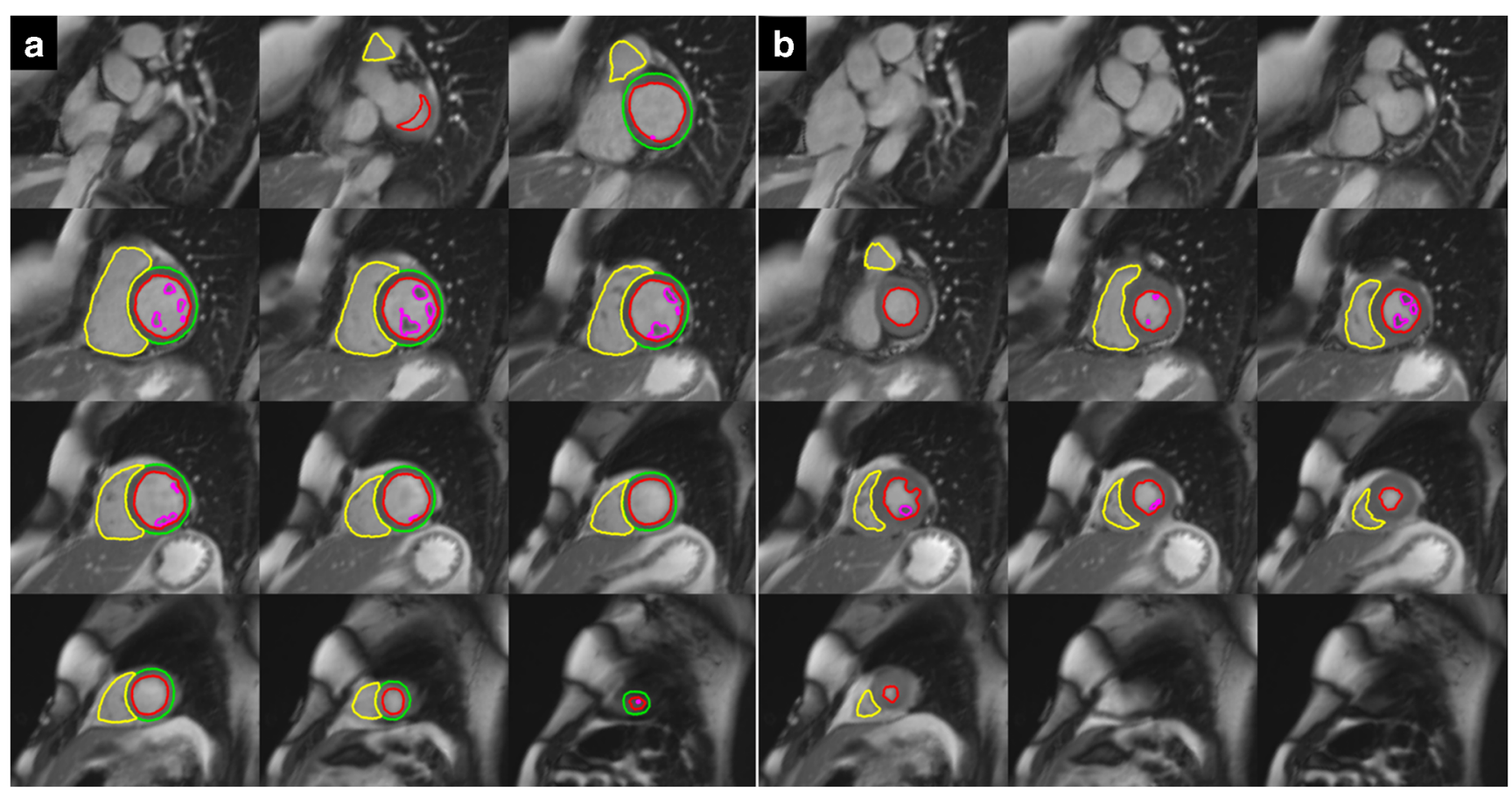

Fig. 1 LV and RV contouring in end-diastole (panel A) and end-systole (panel B) using CMR42 post processing software (Version 5.10.1, Circle Cardiovascular Imaging Inc., Calgary, Canada). Note papillary muscles are included in the left ventricular mass

35). Therefore, patients with ICI-associated myocarditis would be expected to have hyperintensity on T2-weighted imaging. However, the challenge with traditional T2weighted imaging using double or triple inversion recovery turbo spin echo sequence is that the qualitative assessment of the increased signal requires a visual reference of normal myocardium. This is a limitation in this technique when a diffuse process is present, as can be the case with myocarditis, and potentially with ICI-associated myocarditis specifically.
Assessment using quantitative myocardial edema as defined by a T2 myocardium to muscle ratio (T2 ratio) of $>1.9$ can be useful in these patients (47). In a review of 15 patients who underwent CMR at our institution for evaluation of ICI associated myocarditis, qualitative T2-weighted imaging was visually unrevealing in 12 of these patients. However, analysis using quantitative myocardial edema $\mathrm{T} 2$ ratio was noted to have a mean of 2.2 in the lateral and septal wall segments, suggesting more diffuse edema in this disease state (48).
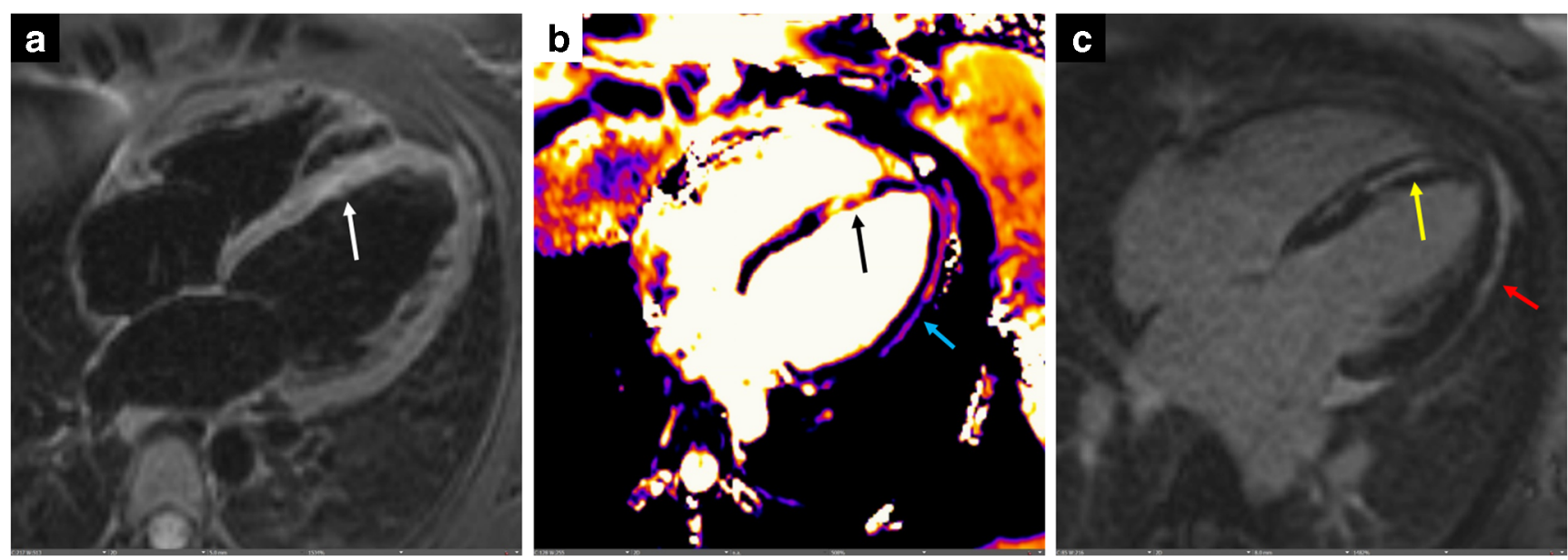

Fig. 2 CMR of a 31-year-old female with fatigue and palpitations, with echocardiogram showing mildly reduced ejection fraction with regional wall motion abnormalities who was referred to our institute given concern for myocarditis. Panel A. 4-chamber view, demonstrating patchy increased signal intensity on $\mathrm{T} 2$-weighted imaging (white arrow), which is better appreciated on T2 mapping as seen in panel B (black arrow) and

post-contrast images demonstrating corresponding region with LGE as seen in panel $\mathrm{C}$ (yellow arrow). Also, increased pericardial signal intensity noted on T2 mapping seen in panel B (blue arrow), which is not well appreciated on the T2-weighted images, and seen on the postcontrast images as well, panel C (red arrow), overall findings suggestive of myopericarditis 
Fig. 3 CMR of a 76-year old male with squamous cell cancer on pembrolizumab demonstrating pericardial thickening and enhancement seen on 4-chamber and short axis view, in panel $\mathrm{A}$ and $\mathrm{B}$ (white arrow), also noted mid myocardial LGE at the inferior RV insertion site seen in panel B (yellow arrow)

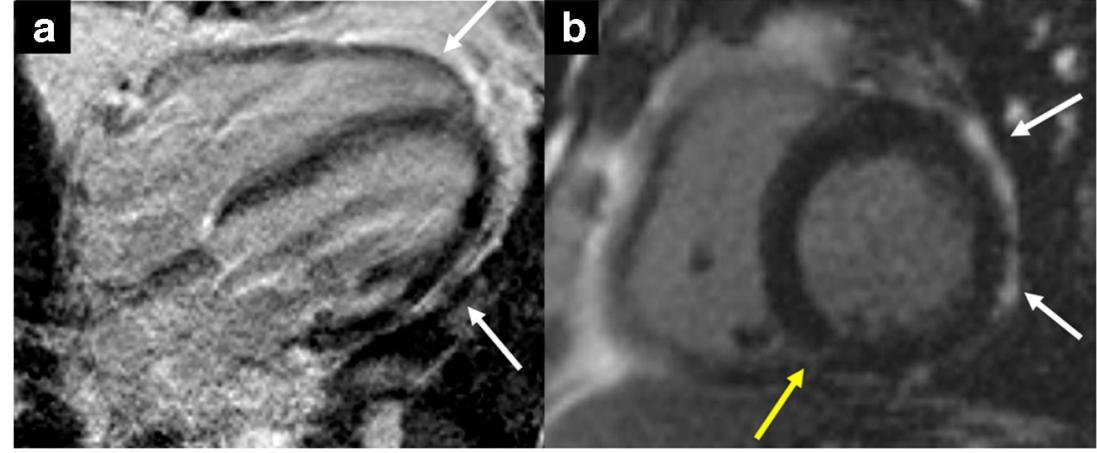

More advanced quantitative methods include parametric mapping, a specialized tool which is highly sensitive and provides quantification of myocardial tissue characteristics on CMR in various disease processes, such as myocarditis, myocardial siderosis, amyloidosis, Anderson-Fabry, anthracycline cardiotoxicity, and various others $(49,50)$. Parametric mapping techniques allow for quantification of myocardial T1 and T2 relaxation values with a high signal to noise ratio and can quantify extracellular volume (ECV) percentage (35). The basic technique for T2 mapping involves acquiring source images with different echo times and plotting the T2 decay curves to generate pixel-wise fitting and a subsequent map (49). A consensus statement by the Society for Cardiovascular Magnetic Resonance endorsed by the European Association for Cardiovascular Imaging on use of parametric mapping provides an excellent guide for its use in the various clinical situations, including myocarditis (49). In myocarditis, T2 mapping can augment significantly the diagnosis of myocardial inflammation, clarifying it's presence or absence, as demonstrated in a myocarditis patient from our institute (Fig. 2). In fact, parametric mapping techniques have been shown to be sensitive for detecting subclinical myocarditis in patients with systemic inflammatory diseases (49, 51-54). Native T1 mapping techniques are also a sensitive indicator for presence of myocardial edema in both acute and chronic myocarditis (35). A study by Ferrira et al. demonstrate that quantification of myocardial native $\mathrm{T} 1$ relaxation values can help detect the location, extent, and patterns of acute myocarditis without the need for contrast (55). There are various T1 mapping sequences available commercially with the modified look-locker inversion recover (MOLLI) or shortened-MOLLI (ShMOLLI) being commonly utilized given the shorter scan times $(43,49)$.

\section{Fibrosis Assessment}

Myocarditis is associated with myocardial inflammation, hyperemia, and subsequently myocardial injury with varying degrees of necrosis and fibrosis, which can be a prognostic indicator for long-term outcomes. CMR can provide tissue characterization, including detection of myocardial early enhancement and late gadolinium enhancement (LGE) after administration of gadolinium-based contrast agents (GBCA). GBCAs are extracellular based, do not enter normal intact myocardial cells, and are cleared, except when there is hyperemia, ruptured cell membranes, or fibrosis, in which case the GBCA is retained and produces high signal as noted on LGE imaging. T1-weighted spin echo imaging after administration of the GBCA can detect early gadolinium enhancement, as it accumulates in the extracellular space, which is increased due to hyperemia (35). Ultimately, fibrosis suggested by the presence of LGE is noted as bright increased signal intensity on delayed imaging obtained 10-15 min after administration of the GBCA. Various patterns of LGE have been recognized in patients with acute myocarditis, including focal areas of midmyocardial to epicardial delayed enhancement. Similarly, in reported cases with ICI-associated myocarditis, the pattern of LGE involvement ranges from mid-myocardial to epicardial, subepicardial, and diffuse LGE $(10,18,56)$. The retrospective review of patients who underwent CMR for concern of ICI myocarditis at our center demonstrated that $75 \%$ revealed LGE in a non-ischemic pattern, with mid-myocardial involvement being the most common pattern (56).

Increase in the myocardial $\mathrm{T} 1$ relaxation values is noted post-contrast on the parametric mapping techniques as the GBCA alters the myocardial T1 relaxation. T1-based criteria for the diagnosis of myocarditis include traditionally used presence of LGE and increased myocardial T1 relaxation times or calculated ECV based on the parametric mapping (35). With parametric mapping techniques, native T1, T2, and ECV have been noted to be elevated in patients with myocarditis (49). ECV is calculated from native T1 mapping and post-contrast T1 mapping values, the patient's hematocrit, and blood pool quantification with a well validated formula (49). A comprehensive review of T1 mapping and ECV by Haaf provides a reference range for normal ECV of around $25 \%$ (57). ECV can be increased in myocarditis and in other cardiomyopathies, including anthracycline induced cardiotoxicity, as demonstrated in a breast cancer patient from our institution $(35,49,50,57)$ (Fig. 4). ECV values have not been reported in ICI-associated myocarditis. However, tissue analysis in ICI-associated myocarditis has demonstrated 

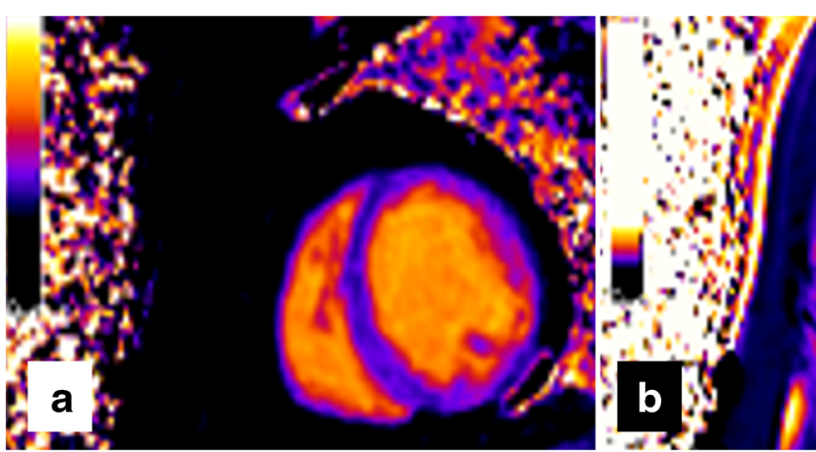

Fig. 4 57-year-old female with history of stage IIA invasive ductal carcinoma of the left breast, status post radiation and anthracycline therapy, who was noted to have a mild drop in the LVEF on echocardiogram and referred for CMR. LVEF was mildly dilated with mildly depressed LVEF at $46 \%$, with mild global hypokinesia and no regional wall motion abnormalities, there was no evidence of LGE on post-contrast images. Parametric mapping was performed given the

inflammatory infiltrate, mostly leucocytic, with an increase in extracellular volume, suggesting that this technique may be helpful in this disease state (58).

\section{Myocardial Strain Imaging}

Myocardial strain is the deformation of the myocardium from the initial state in end-diastole compared to systole, expressed as a percentage, which can be measured in longitudinal, circumferential, and radial dimensions and has been used in trans-thoracic echocardiography (TTE) to detect preclinical and subtle changes in LV function before a drop in the LVEF (59). Global longitudinal strain (GLS) assessment by TTE has been used in the diagnosis and follow up of patients with chemotherapy-induced cardiotoxicity (60) and has been incorporated into screening guidelines (61). There is very limited published data related to the use of TTE GLS in patients with ICI-associated myocarditis. Reduced TTE GLS has been noted in patients with ICI-associated myocarditis with either normal or reduced LVEF and was associated with increased MACE (65).
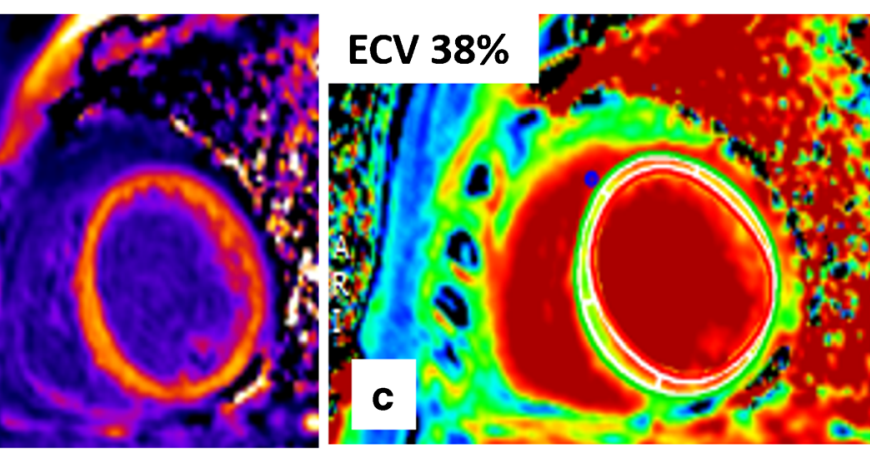

concern for anthracycline related cardiotoxicity, which showed increased ECV at $38 \%$ consistent with the diagnosis. Panel A showing native T1 mapping and Panel B showing post contrast T1 mapping, performed using ShMOLLI technique, post processing was performed on CMR42 post processing software (Version 5.10.1, Circle Cardiovascular Imaging Inc., Calgary, Canada) for generating ECV map (panel C) and values

However, limitations with TTE strain include the need for good image quality, which is dependent on patient factors, including acoustic windows, and inter-vendor variability. Myocardial strain assessment by CMR can overcome some of these patient factors; however, there is variability in techniques for obtaining myocardial strain by CMR as well. There are various methods including CMR tagging, CMR feature tracking (CMR-FT), displacement encoding with simulated echos (DENSE), and strain encoding (SENC) imaging, all of which have their own limitations $(59,62)$. CMR-FT is similar to the TTE speckle tracking method, where it identifies and tracks the features in the image. CMR-FT method does not require additional sequences or acquisitions and can be calculated by post-processing the cine SAS and long axis sequences by various commercially available software. Reduction in CMR circumferential strain (CS) was noted in patients after anthracycline exposure, which was predictive of an early preclinical drop in LVEF (63). Based on our experience with a retrospective study of breast cancer patients, CMR-FT GLS demonstrated a moderate correlation with TTE GLS (64) (Fig. 5). SENC imaging utilizes magnetization tags to detect myocardial compression and is a separately acquired CMR
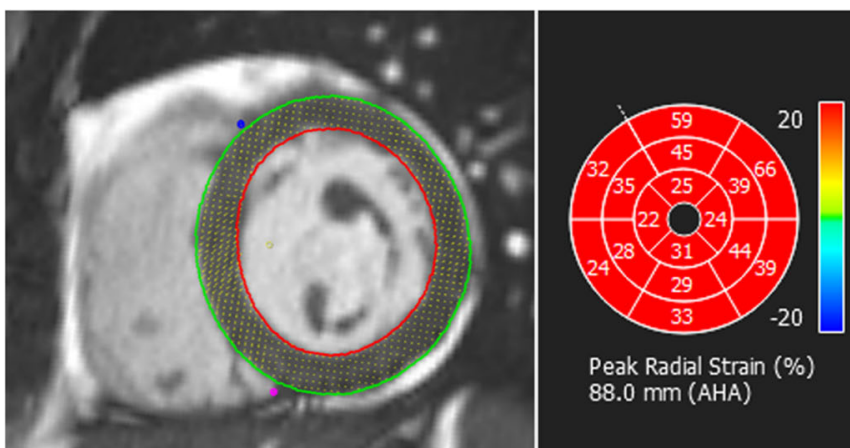

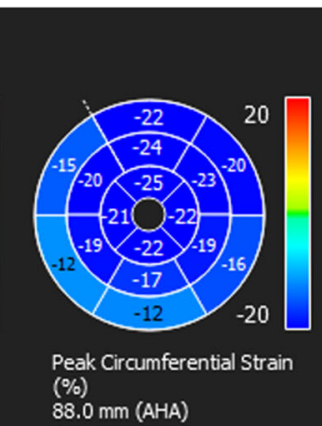

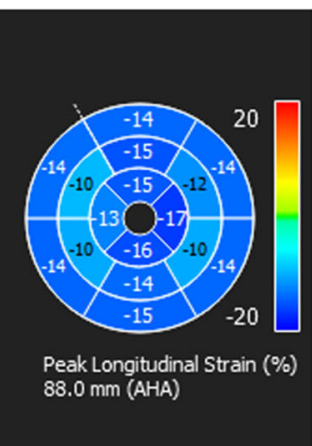

Fig. 5 Example of feature tracking using CMR42 post processing software (Version 5.10.1, Circle Cardiovascular Imaging Inc., Calgary, Canada), endocardium (red) and epicardium (green) contours manually traced on the SSFP cine short axis stack, long axis series. The yellow dots between the red and green contours correspond to the myocardial points which are tracked during systole in feature tracking strain computation 
Table 1 Recommended protocol/sequences for suspected ICI associated myocarditis

\begin{tabular}{|c|c|}
\hline Sequence & Assessment \\
\hline Cine short axis stack and long axis views (2-chamber, 3-chamber, 4-chamber) & $\begin{array}{l}\text { LV and RV structure and function, regional wall motion, LV mass, } \\
\text { pericardial structure, LV myocardial strain }\end{array}$ \\
\hline $\begin{array}{l}\text { Myocardial tagging and real-time cine with deep inspiration if suspected } \\
\text { pericarditis or constriction }\end{array}$ & Pericardial disease and constriction \\
\hline T2-weighted images, preferably with fat saturation & Myocardial or pericardial edema/inflammation \\
\hline Short axis views, (2-chamber, 3-chamber, 4-chamber) & If appears global or negative, then consider $\mathrm{T} 2$ ratio \\
\hline $\begin{array}{l}\text { T2 mapping, if available then do not need to perform T2-weighted imaging } \\
\text { Short axis views, (2-chamber, 3-chamber, 4-chamber) }\end{array}$ & Myocardial or pericardial edema/inflammation \\
\hline $\begin{array}{l}\text { T1 mapping pre-contrast } \\
\text { Short axis views }\end{array}$ & Myocardial edema/inflammation and fibrosis \\
\hline $\begin{array}{l}\text { T1 mapping post-contrast } \\
\text { Short axis views }\end{array}$ & Myocardial ECV quantification \\
\hline $\begin{array}{l}\text { Late gadolinium imaging } \\
\text { Short axis stack, 2-chamber, 3-chamber, 4-chamber) }\end{array}$ & Myocardial or pericardial edema/inflammation and fibrosis \\
\hline
\end{tabular}

sequence which requires post-processing with their proprietary software (Myostrain software, Myocardial solutions, Morrisville, North Carolina). SENC imaging is being currently used in a clinical trial for early diagnosis of chemotherapyinduced cardiotoxicity (https://clinicaltrials.gov/ct2/show/ NCT03543228). CMR myocardial strain information, in conjunction with LVEF and tissue characterization, could also potentially be used in the future for risk stratification of patients with ICI myocarditis and help guide need for early aggressive management in high-risk patients.

\section{Prognosis and Surveillance}

CMR is not only an invaluable tool for the diagnosis of myocarditis in general, but also it is an excellent tool for providing prognostic information in these patients (66). LVEF and myocardial strain have been utilized in the field of cardio-oncology for monitoring cardiotoxicity from chemotherapy agents, such as anthracyclines (60). However, as noted in various case reports and case series, the LVEF in ICI-associated myocarditis can be normal or reduced. Interesting, a reduced LVEF was not associated with increased MACE in ICI-associated myocarditis, according to a recent review of 35 patients from a multicenter registry (10). In the retrospective review of patients who underwent CMR for concern for ICI myocarditis at our center, $75 \%$ of patients were diagnosed with and treated for ICI-associated myocarditis, and $58 \%$ of patients had recovery of LVEF (56). In a retrospective study of 668 patients with suspected myocarditis by Gräni et al., with a median follow up of 4.7 years, presence of LGE was associated with a more than double the risk of major cardiovascular adverse events with a hazard ratio 2.22 (95\% confidence interval: 1.47 to $3.35, p<0.001$ ) (66). Gräni et al. performed a comprehensive study of patients with myocarditis using various methods for scar quantification including $2 \mathrm{SD}, 3 \mathrm{SD}, 4 \mathrm{SD}, 5 \mathrm{SD}, 6 \mathrm{SD}$, and full width half maximum (FWHM) (67). They showed that scar quantification using full width half maximum method, followed by visual LGE scoring, can be used for risk stratification in patients with myocarditis and predict MACE (67). Similarly, scar quantification by CMR could be utilized in risk stratification of patients with ICI associated myocarditis. Parametric mapping can also be a helpful prognostic tool as demonstrated on a recent study by Grani et al., which showed a significantly increased MACE associated with an ECV $>35 \%$ in myocarditis patients (68). Myocardial strain is not well studied and reported in patients with ICI-associated myocarditis but has potential for detecting a preclinical reduction in LVEF and could be part of a CMR evaluation for screening and monitoring patients with suspected ICI-associated myocarditis.

\section{Conclusions}

With the knowledge from the reported cases of ICI-associated myocarditis in the literature, and based on experience at our own institution, the clinical presentation can be quite variable, ranging from mild to fulminant fatal myocarditis. Furthermore, the traditional clinical adage of viral myocarditis with troponin elevation, ECG changes, and focal myocardial involvement, as noted on CMR, is not always seen in ICI associated myocarditis. There have been reported cases of ICI-associated myocarditis with no elevation of cardiac biomarkers, normal LVEF, and nonspecific ECG changes, making the diagnosis even more challenging, although it is unclear how many patients with lymphocytic infiltrate on biopsy with other negative findings would be expected to progress to a more severe form of myocarditis. CMR is an imaging tool with high sensitivity and specificity for assessment of LV 
and RV function, regional wall motion, presence of myocardial edema, detection of LGE, and ECV quantification, all helping to bridge the gap in making an accurate diagnosis. Such a proposed comprehensive CMR acquisition protocol is referred to in Table 1. Currently, there is a lack of established values for native T1, T2 and ECV in ICI associated myocarditis, though these could be established in the future with increased use of parametric mapping in these patients. Overall, CMR carries the advantage of being a comprehensive noninvasive modality for diagnosis of ICI-associated myocarditis and holds promise for screening and serial follow up in patients exposed to ICIs.

Acknowledgment This work was supported by American Heart Association grant \#18CDA34110361/Lauren A. Baldassarre, MD/2018

\section{Compliance with Ethical Standards}

Conflict of Interest The authors declare that they have no conflict of interest.

Human and Animal Rights and Informed Consent This article does not contain any studies with human or animal subjects performed by any of the authors.

Open Access This article is licensed under a Creative Commons Attribution 4.0 International License, which permits use, sharing, adaptation, distribution and reproduction in any medium or format, as long as you give appropriate credit to the original author(s) and the source, provide a link to the Creative Commons licence, and indicate if changes were made. The images or other third party material in this article are included in the article's Creative Commons licence, unless indicated otherwise in a credit line to the material. If material is not included in the article's Creative Commons licence and your intended use is not permitted by statutory regulation or exceeds the permitted use, you will need to obtain permission directly from the copyright holder. To view a copy of this licence, visit http://creativecommons.org/licenses/by/4.0/.

\section{References}

Papers of particular interest, published recently, have been highlighted as:

- Of importance

•• Of major importance

1. De Velasco G, Je Y, Bosse D, Awad MM, Ott PA, Moreira RB, et al. Comprehensive meta-analysis of key immune-related adverse events from CTLA-4 and PD-1/PD-L1 inhibitors in Cancer patients. Cancer Immunol Res. 2017;5(4):312-8.

2. Koelzer VH, Rothschild SI, Zihler D, Wicki A, Willi B, Willi N, et al. Systemic inflammation in a melanoma patient treated with immune checkpoint inhibitors-an autopsy study. J Immunother Cancer. 2016;4:13.

3. Tajiri K, Ieda M. Cardiac complications in immune checkpoint inhibition therapy. Front Cardiovasc Med. 2019;6:3.

4. Palmieri DJ, Carlino MS. Immune checkpoint inhibitor toxicity. Curr Oncol Rep. 2018;20(9):72.
5. Yun S, Vincelette ND, Mansour I, Hariri D, Motamed S. Late onset ipilimumab-induced pericarditis and pericardial effusion: a rare but life threatening complication. Case Rep Oncol Med. 2015;2015: 794842.

6. Sharma M, Suero-Abreu GA, Kim B. A case of acute heart failure due to immune checkpoint blocker Nivolumab. Cardiol Res. 2019;10(2):120-3.

7. Agrawal N, Khunger A, Vachhani P, Colvin TA, Hattoum A, Spangenthal E, et al. Cardiac toxicity associated with immune checkpoint inhibitors: case series and review of the literature. Case Rep Oncol. 2019;12(1):260-76.

8. Atallah-Yunes SA, Kadado AJ, Kaufman GP, Hernandez-Montfort J. Immune checkpoint inhibitor therapy and myocarditis: a systematic review of reported cases. J Cancer Res Clin Oncol. 2019;145(6):1527-57.

9. Salem J-E, Manouchehri A, Moey M, Lebrun-Vignes B, Bastarache L, Pariente A, et al. Cardiovascular toxicities associated with immune checkpoint inhibitors: an observational, retrospective, pharmacovigilance study. The Lancet Oncology. 2018;19(12): 1579-89.

10. Mahmood SS, Fradley MG, Cohen JV, Nohria A, Reynolds KL, Heinzerling LM, et al. Myocarditis in patients treated with immune checkpoint inhibitors. J Am Coll Cardiol. 2018;71(16):1755-64.

11. Wang J, Okazaki IM, Yoshida T, Chikuma S, Kato Y, Nakaki F, et al. PD-1 deficiency results in the development of fatal myocarditis in MRL mice. Int Immunol. 2010;22(6):443-52.

12. Ganatra S, Neilan TG. Immune checkpoint inhibitor-associated myocarditis. Oncologist. 2018;23(8):879-86.

13. Raikhelkar J, Uriel N. Immune checkpoint inhibitor myocarditis. Curr Opin Cardiol. 2019;34(3):303-6.

14. Bajwa R, Cheema A, Khan T, Amirpour A, Paul A, Chaughtai S, et al. Adverse effects of immune checkpoint inhibitors (programmed Death-1 inhibitors and cytotoxic T-lymphocyteassociated Protein-4 inhibitors): results of a retrospective study. J Clin Med Res. 2019;11(4):225-36.

15. Norwood TG, Westbrook BC, Johnson DB, Litovsky SH, Terry NL, McKee SB, et al. Smoldering myocarditis following immune checkpoint blockade. J Immunother Cancer. 2017;5(1):91.

16. Lauer B, Niederau C, Kuhl U, Schannwell M, Pauschinger M, Strauer BE, et al. Cardiac troponin $\mathrm{T}$ in patients with clinically suspected myocarditis. J Am Coll Cardiol. 1997;30(5):1354-9.

17. Kindermann I, Barth C, Mahfoud F, Ukena C, Lenski M, Yilmaz A, et al. Update on myocarditis. J Am Coll Cardiol. 2012;59(9):77992.

18. Gallegos C, Rottmann D, Nguyen VQ, Baldassarre LA. Myocarditis with checkpoint inhibitor immunotherapy: case report of late gadolinium enhancement on cardiac magnetic resonance with pathology correlate. Eur Heart J Case Rep. 2019;3(1):yty149.

19. Heinzerling L, Ott PA, Hodi FS, Husain AN, Tajmir-Riahi A, Tawbi $\mathrm{H}$, et al. Cardiotoxicity associated with CTLA4 and PD1 blocking immunotherapy. J Immunother Cancer. 2016;4:50.

20. Lee Chuy K, Oikonomou EK, Postow MA, Callahan MK, Chapman PB, Shoushtari AN, et al. Myocarditis surveillance in patients with advanced melanoma on combination immune checkpoint inhibitor therapy: the memorial Sloan Kettering Cancer center experience. Oncologist. 2019;24(5):e196-e7.

21. Behling J, Kaes J, Munzel T, Grabbe S, Loquai C. New-onset thirddegree atrioventricular block because of autoimmune-induced myositis under treatment with anti-programmed cell death-1 (nivolumab) for metastatic melanoma. Melanoma Res. 2017;27(2):155-8.

22. Reddy N, Moudgil R, Lopez-Mattei JC, Karimzad K, Mouhayar EN, Somaiah N, et al. Progressive and reversible conduction disease with checkpoint inhibitors. Can J Cardiol. 2017;33(10):1335 e13-5. 
23. Ederhy S, Cautela J, Ancedy Y, Escudier M, Thuny F, Cohen A. Takotsubo-like syndrome in Cancer patients treated with immune checkpoint inhibitors. JACC Cardiovasc Imaging. 2018;11(8): 1187-90.

24. Hauck AJ, Kearney DL, Edwards WD. Evaluation of postmortem endomyocardial biopsy specimens from 38 patients with lymphocytic myocarditis: implications for role of sampling error. Mayo Clin Proc. 1989;64(10):1235-45.

25. Cooper LT, Baughman KL, Feldman AM, Frustaci A, Jessup M, Kuhl U, et al. The role of endomyocardial biopsy in the management of cardiovascular disease: a scientific statement from the American Heart Association, the American College of Cardiology, and the European Society of Cardiology. Endorsed by the Heart Failure Society of America and the heart failure Association of the European Society of cardiology. J Am Coll Cardiol. 2007;50(19):1914-31.

26. Tadokoro T, Keshino E, Makiyama A, Sasaguri T, Ohshima K, Katano H, et al. Acute lymphocytic myocarditis with anti-PD-1 antibody nivolumab. Circ Heart Fail. 2016;9(10).

27. Imai R, Ono M, Nishimura N, Suzuki K, Komiyama N, Tamura T. Fulminant myocarditis caused by an immune checkpoint inhibitor: a case report with pathologic findings. J Thorac Oncol. 2019;14(2): e36-e8.

28. Rota E, Varese P, Agosti S, Celli L, Ghiglione E, Pappalardo I, et al. Concomitant myasthenia gravis, myositis, myocarditis and polyneuropathy, induced by immune-checkpoint inhibitors: a lifethreatening continuum of neuromuscular and cardiac toxicity. eNeurologicalSci. 2019;14:4-5.

29. Jain V, Bahia J, Mohebtash M, Barac A. Cardiovascular complications associated with novel Cancer immunotherapies. Curr Treat Options Cardiovasc Med. 2017;19(5):36.

30. Tay RY, Blackley E, McLean C, Moore M, Bergin P, Gill S, et al. Successful use of equine anti-thymocyte globulin (ATGAM) for fulminant myocarditis secondary to nivolumab therapy. Br $\mathrm{J}$ Cancer. 2017;117(7):921-4.

31. Brahmer JR, Lacchetti C, Schneider BJ, Atkins MB, Brassil KJ, Caterino JM, et al. Management of Immune-Related Adverse Events in patients treated with immune checkpoint inhibitor therapy: American Society of Clinical Oncology clinical practice guideline. J Clin Oncol. 2018;36(17):1714-68.

32. Moslehi JJ, Salem J-E, Sosman JA, Lebrun-Vignes B, Johnson DB. Increased reporting of fatal immune checkpoint inhibitor-associated myocarditis. Lancet. 2018;391(10124):933.

33. American College of Cardiology Foundation Task Force on Expert, Consensus D, Hundley WG, Bluemke DA, Finn JP, Flamm SD, et al. ACCF/ACR/AHA/NASCI/SCMR 2010 expert consensus document on cardiovascular magnetic resonance: a report of the American College of Cardiology Foundation task force on expert consensus documents. J Am Coll Cardiol. 2010;55(23):2614-62.

34. Friedrich MG, Sechtem U, Schulz-Menger J, Holmvang G, Alakija P, Cooper LT, et al. Cardiovascular magnetic resonance in myocarditis: a JACC white paper. J Am Coll Cardiol. 2009;53(17):1475-87.

35. Ferreira VM, Schulz-Menger J, Holmvang G, Kramer CM, Carbone I, Sechtem U, et al. Cardiovascular magnetic resonance in nonischemic myocardial inflammation: expert recommendations. J Am Coll Cardiol. 2018;72(24):3158-76.

36. Altan M, Toki MI, Gettinger SN, Carvajal-Hausdorf DE, Zugazagoitia J, Sinard JH, et al. Immune checkpoint inhibitorassociated pericarditis. J Thorac Oncol. 2019;14(6):1102-8.

37. Nieminen MS, Heikkila J, Karjalainen J. Echocardiography in acute infectious myocarditis: relation to clinical and electrocardiographic findings. Am J Cardiol. 1984;53(9):1331-7.

38. Felker GM, Boehmer JP, Hruban RH, Hutchins GM, Kasper EK, Baughman KL, et al. Echocardiographic findings in fulminant and acute myocarditis. J Am Coll Cardiol. 2000;36(1):227-32.
39. Bellenger NG, Grothues F, Smith GC, Pennell DJ. Quantification of right and left ventricular function by cardiovascular magnetic resonance. Herz. 2000;25(4):392-9.

40. Barkhausen J, Ruehm SG, Goyen M, Buck T, Laub G, Debatin JF. MR evaluation of ventricular function: true fast imaging with steady-state precession versus fast low-angle shot cine MR imaging: feasibility study. Radiology. 2001;219(1):264-9.

41. Moon JC, Lorenz CH, Francis JM, Smith GC, Pennell DJ. Breathhold FLASH and FISP cardiovascular MR imaging: left ventricular volume differences and reproducibility. Radiology. 2002;223(3): 789-97.

42. Gandy SJ, Waugh SA, Nicholas RS, Simpson HJ, Milne W, Houston JG. Comparison of the reproducibility of quantitative cardiac left ventricular assessments in healthy volunteers using different MRI scanners: a multicenter simulation. J Magn Reson Imaging. 2008;28(2):359-65.

43. Kawel-Boehm N, Maceira A, Valsangiacomo-Buechel ER, VogelClaussen J, Turkbey EB, Williams R, et al. Normal values for cardiovascular magnetic resonance in adults and children. $\mathrm{J}$ Cardiovasc Magn Reson. 2015;17:29.

44. Vogel-Claussen J, Finn JP, Gomes AS, Hundley GW, JeroschHerold M, Pearson G, et al. Left ventricular papillary muscle mass: relationship to left ventricular mass and volumes by magnetic resonance imaging. J Comput Assist Tomogr. 2006;30(3):426-32.

45. Zagrosek A, Wassmuth R, Abdel-Aty H, Rudolph A, Dietz R, Schulz-Menger J. Relation between myocardial edema and myocardial mass during the acute and convalescent phase of myocarditis-a CMR study. J Cardiovasc Magn Reson. 2008;10:19.

46. Eitel I, Friedrich MG. T2-weighted cardiovascular magnetic resonance in acute cardiac disease. J Cardiovasc Magn Reson. 2011;13: 13.

47. Carbone I, Childs H, Aljizeeri A, Merchant N, Friedrich MG. Importance of reference muscle selection in quantitative signal intensity analysis of T2-weighted images of myocardial edema using a T2 ratio method. Biomed Res Int. 2015;2015:232649.

48. Soufer A, Higgins A, Arbune A, Baldassarre LA. Quantitative analysis of T2 imaging may identify global edema in patients referred for immune checkpoint inhibitor cardiomyopathy. Society of Cardiac Magnetic Resonance; Feb 2019 Bellvue, WA 2019.

49. Messroghli DR, Moon JC, Ferreira VM, Grosse-Wortmann L, He $\mathrm{T}$, Kellman P, et al. Clinical recommendations for cardiovascular magnetic resonance mapping of $\mathrm{T} 1, \mathrm{~T} 2, \mathrm{~T} 2 *$ and extracellular volume: a consensus statement by the Society for Cardiovascular Magnetic Resonance (SCMR) endorsed by the European Association for Cardiovascular Imaging (EACVI). J Cardiovasc Magn Reson. 2017;19(1):75.

50. Neilan TG, Coelho-Filho OR, Shah RV, Feng JH, Pena-Herrera D, Mandry D, et al. Myocardial extracellular volume by cardiac magnetic resonance imaging in patients treated with anthracyclinebased chemotherapy. Am J Cardiol. 2013;111(5):717-22.

51. Ntusi NA, Piechnik SK, Francis JM, Ferreira VM, Rai AB, Matthews PM, et al. Subclinical myocardial inflammation and diffuse fibrosis are common in systemic sclerosis-a clinical study using myocardial T1-mapping and extracellular volume quantification. J Cardiovasc Magn Reson. 2014;16:21.

52. Ntusi NAB, Piechnik SK, Francis JM, Ferreira VM, Matthews PM, Robson MD, et al. Diffuse myocardial fibrosis and inflammation in rheumatoid arthritis: insights from CMR T1 mapping. JACC Cardiovasc Imaging. 2015;8(5):526-36.

53. Puntmann VO, D'Cruz D, Smith Z, Pastor A, Choong P, Voigt T, et al. Native myocardial T1 mapping by cardiovascular magnetic resonance imaging in subclinical cardiomyopathy in patients with systemic lupus erythematosus. Circ Cardiovasc Imaging. 2013;6(2):295-301. 
54. Crouser ED, Ono C, Tran T, He X, Raman SV. Improved detection of cardiac sarcoidosis using magnetic resonance with myocardial T2 mapping. Am J Respir Crit Care Med. 2014;189(1):109-12.

55. Ferreira VM, Piechnik SK, Dall'Armellina E, Karamitsos TD, Francis JM, Ntusi N, et al. Native T1-mapping detects the location, extent and patterns of acute myocarditis without the need for gadolinium contrast agents. J Cardiovasc Magn Reson. 2014;16:36.

56. Higgins A, Soufer A, Nguyen V, Boutagy NE, Gallegos C, Charifa $\mathrm{A}$, et al. Cardiac magnetic resonance imaging aids in diagnosis of suspected immune checkpoint inhibitor mediated cardiotoxicity. Global Cardio-Oncology Summit; Sept 2018. Tampa; 2018.

57. Haaf P, Garg P, Messroghli DR, Broadbent DA, Greenwood JP, Plein S. Cardiac T1 mapping and extracellular volume (ECV) in clinical practice: a comprehensive review. J Cardiovasc Magn Reson. 2016;18(1):89.

58. Upadhrasta S, Elias H, Patel K, Zheng L. Managing cardiotoxicity associated with immune checkpoint inhibitors. Chronic Dis Transl Med. 2019;5(1):6-14.

59. Scatteia A, Baritussio A, Bucciarelli-Ducci C. Strain imaging using cardiac magnetic resonance. Heart Fail Rev. 2017;22(4):465-76.

60. Thavendiranathan P, Poulin F, Lim KD, Plana JC, Woo A, Marwick $\mathrm{TH}$. Use of myocardial strain imaging by echocardiography for the early detection of cardiotoxicity in patients during and after cancer chemotherapy: a systematic review. J Am Coll Cardiol. 2014;63(25 Pt A):2751-68.

61. Plana JC, Galderisi M, Barac A, Ewer MS, Ky B, Scherrer-Crosbie $\mathrm{M}$, et al. Expert consensus for multimodality imaging evaluation of adult patients during and after cancer therapy: a report from the American Society of Echocardiography and the European Association of Cardiovascular Imaging. J Am Soc Echocardiogr. 2014;27(9):911-39.

62. Osman NF. Detecting stiff masses using strain-encoded (SENC) imaging. Magn Reson Med. 2003;49(3):605-8.
63. Jolly MP, Jordan JH, Melendez GC, McNeal GR, D'Agostino RB Jr, Hundley WG. Automated assessments of circumferential strain from cine CMR correlate with LVEF declines in cancer patients early after receipt of cardio-toxic chemotherapy. J Cardiovasc Magn Reson. 2017;19(1):59.

64. Arbune A, Kwan J, Hennery M, Baldassarre LA. Correlation of myocardial strain imaging by cardiac magnetic resonance imaging with echocardiography in breast cancer patients with concern for cardiotoxicity. Society of Cardiac Magnetic Resonance; Feb 2019 Bellvue, WA 2019.

65. Awadalla M, Mahmood S, Groarke J, Liu S, Hassan MZO, Murphy SPT, et al. Decreased global longitudinal strain with myocarditis from immune checkpoint inhibitors and occurrence of major adverse cardiac events. J Am Coll Cardiol. 2019;73(9).

66. Grani C, Eichhorn C, Biere L, Murthy VL, Agarwal V, Kaneko K, et al. Prognostic value of cardiac magnetic resonance tissue characterization in risk stratifying patients with suspected myocarditis. J Am Coll Cardiol. 2017;70(16):1964-76.

67. Grani C, Eichhorn C, Biere L, Kaneko K, Murthy VL, Agarwal V, et al. Comparison of myocardial fibrosis quantification methods by cardiovascular magnetic resonance imaging for risk stratification of patients with suspected myocarditis. J Cardiovasc Magn Reson. 2019;21(1):14.

68. Grani C, Biere L, Eichhorn C, Kaneko K, Agarwal V, Aghayev A, et al. Incremental value of extracellular volume assessment by cardiovascular magnetic resonance imaging in risk stratifying patients with suspected myocarditis. Int J Card Imaging. 2019;35(6):106778 .

Publisher's Note Springer Nature remains neutral with regard to jurisdictional claims in published maps and institutional affiliations. 